\title{
Fibrous Web in the Right Atrium: A Complication of Fungal Endocarditis and Previous Lead Infections
}

\author{
Harry Wang, MD
}

\section{CASE PRESENTATION}

A 57-year-old woman with a past medical history of severe non-ischemic cardiomyopathy undergoing orthotopic heart transplant evaluation on milrinone, prior extraction of multiple pacemaker and implantable cardioverter defibrillator (ICD) leads due to Staphylococcus epidermidis endocarditis, and multiple central venous catheter infections presented with several days of worsening dyspnea on exertion, orthopnea and lower extremity edema, prompting admission for congestive heart failure exacerbation.

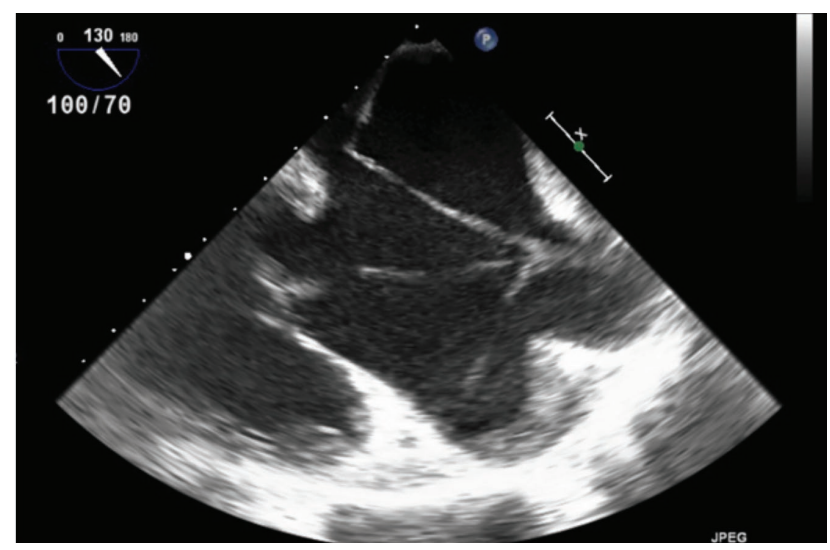

Figure 1: Linear vegetations extending to the coronary sinus and right atrial appendage. Middle linear echodensity is the current lead.

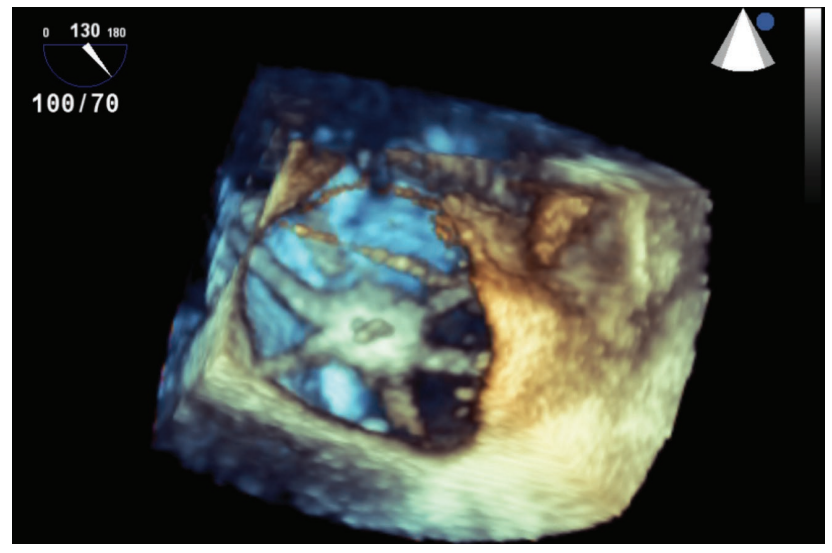

Figure 2: 3D view on TEE of vegetation on the pacemaker lead with multiple linear echodensities emanating from the lead.
While she received aggressive intravenous diuretics, she was also found to be febrile to $101.8^{\circ} \mathrm{F}$ with a white blood cell count of $11,000 / \mu \mathrm{L}$. Blood cultures were drawn, her peripherally inserted central catheter was removed, and she was started on broad spectrum antibiotics with imipenem and daptomycin. She remained hemodynamically stable, but several days into her hospitalization, her blood cultures grew Candida albicans. Antibiotics were stopped, and she was empirically started on anidulafungin. A transesophageal echocardiogram (TEE) was then performed and showed linear densities in the right atrium which mimicked the course of previously extracted leads (Figure 1), consistent with fibrous sheaths. Also seen was a $5 \times 8 \mathrm{~mm}$ mobile vegetation on the ICD lead, multiple linear 'web-like' vegetations in the right atrium (Figure 2), and more typical-appearing vegetations in the superior vena cava (Figure 3). These findings, in addition to the fungemia, prompted transvenous ICD extraction, which she tolerated well. Her subsequent blood cultures were negative, but given her previous history of lead and line infections, the decision was made to fit the patient with a wearable defibrillator and re-evaluate her for infection in 6 weeks after completion of treatment for fungal endocarditis. After clearance of infection, her candidacy for cardiac transplant would also be re-evaluated.

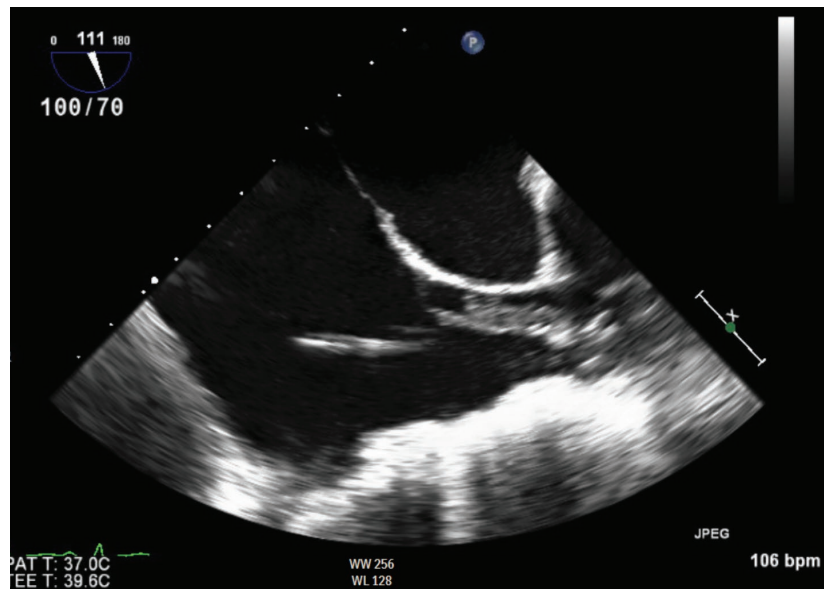

Figure 3: TEE showing a large SVC Vegetation extending into the right atrium. 


\section{DISCUSSION}

ICD infections often necessitate transvenous lead extraction to prevent significant mortality; however, in a minority of cases, intracardiac 'sheaths' or 'casts' can be seen on echocardiography after lead extraction. ${ }^{1}$ ICD leads can rarely be encapsulated by fibrous sheaths which grow over time and remain even after lead extraction. The incidence of residual fibrocalcific tissue is estimated to be around $8-14 \% .^{2}$ These sheaths are postulated to be a potential nidus for endocarditis, and their presence is associated with a higher risk of death after lead extraction. ${ }^{3}$ Clinicians caring for patients with cardiovascular implantable electronic devices need to be aware of this entity as their presence may affect further management. Although they can be detected by TEE, the entire clinical setting must be carefully examined to determine which patients with these fibrous sheaths may have endocarditis and require more diligent follow up.

\section{REFERENCES}

1. Caiati, C., Pollice, P., Lepera, M. E., Luzzi, G. and Favale, S. An Insight Into The Etiology Of New Intracardiac Masses (Ghost) After Infected Lead Extraction: A Single-Center Study Performed By Intracardiac Echocardiography. Journal of the American College of Cardiology. 2018; 71: 30899-4.

2. Kiuchi, K. et al. The details of an unusual "ghost" after transvenous lead extraction: Three-dimensional computed tomography analysis. Journal of Arrhythmia. 2017; 33: 640-642.

3. Narducci, M. L. et al. Presence of 'ghosts' and mortality after transvenous lead extraction. Europace. 2017; 19(3): 432-440. 